\title{
Postmonolingual schreiben?
}

\section{Zum Jargon der Philologie}

Till Dembeck / Georg Mein

Es gibt eine Nachreife auch der festgelegten Worte.

Walter Benjamin: Die Aufgabe des Übersetzers

In Berufsgruppen, die, wie das so heißt, geistige Arbeit verrichten, zugleich aber unselbständig und abhängig sind oder wirtschaftlich schwach, ist der Jargon Berufskrankheit.

Theodor W. Adorno: Jargon der Eigentlichkeit

Das Einsprachigkeitsparadigma, also die Vorstellung, dem einzelnen Menschen sei eine, nämlich seine Sozialisationssprache eigen und diese eine Sprache biete ihm naturgemäß die besten Ausdrucksmöglichkeiten, wird zunehmend zerbrechlich. $\mathrm{Zu}$ Recht wird darauf verwiesen, dass es sich um eine westliche Erfindung der Neuzeit handelt, die im Namen des >nation building kulturelle Homogenität und einen einheitlichen Kommunikationsraum herstellen soll. Für den deutschen Sprachraum ist diese Verbindung von Sprache und Nation insbesondere mit dem Namen Jacob Grimm verbunden, der sie in vielen seiner Schriften immer wieder emphatisch beschwört, wie etwa in seiner AkademieSchrift Über den Ursprung der Sprache, wo es heißt: »[D]ie kraft der sprache bildet völker und hält sie zusammen, ohne ein solches band würden sie sich versprengen« (Grimm 1858: 30). Doch wenn dieses Paradigma der Einsprachigkeit nicht mehr greift, was tritt dann an seine Stelle?

Unter den Kritikern des Einsprachigkeitsparadigmas scheint Einigkeit darüber zu herrschen, dass die umstandslose Rückkehr zu einer >ursprünglichen< Mehrsprachigkeit nicht möglich ist. Yildiz spricht aktuell von einer »postmonolingual condition « und signalisiert damit Unsicherheit darüber, was an die Stelle der Einsprachigkeit zu setzen wäre (Yildiz 2012). Die Soziolinguisten Makoni und Pennycook legen dar, dass Mehrsprachigkeit nicht schlicht die Pluralisierung von Einsprachigkeiten sein dürfe, wolle sie das Einsprachigkeitspara- 
digma überwinden; ${ }^{1}$ stattdessen müsse es um das »disinventing « sprachlicher Einheitlichkeit gehen - man könnte mit einer von Stockhammer und anderen vorgeschlagenen Kategorie auch sagen, man müsse die einfache >Sprachigkeit< sprachlicher Elemente und Strukturen, also ihre Zuordenbarkeit zu einer Sprache (im Sinne von Langue), zerbrechlich machen (Stockhammer/Arndt/Naguschewski 2007).

Die folgenden Ausführungen widmen sich den Alternativen des Einsprachigkeitsparadigmas an einem Beispiel, das uns als Philologen - im emphatischen Sinne des Wortes - selbst angeht: Er nimmt >unsere< Sprache in den Blick und fragt nach Möglichen eines post-monolingualen philologischen Schreibens. Dass diese Frage relevant ist und sich keinesfalls nur aus sprachhistorischer Perspektive aufdrängt, sondern auch allgemeine kulturhistorische Bedeutung hat, zeigt etwa die Arbeit von Jürgen Fohrmann Das Projekt der deutschen Literaturgeschichte, die luzide die Bemühungen der sich konstituierenden Nationalphilologie Germanistik vom Ende des 18. bis zum Ende des 19. Jahrhunderts vor Augen führt, die Geschichte der deutschen Literatur als Entelechie eines Nationalcharakters zu beschreiben (Fohrmann 1989).

Wir werden uns im Folgenden zunächst mit den Gründen für die Wirkmächtigkeit des Einsprachigkeitsparadigmas auseinandersetzen - in der Philologie und anderswo (Abschn. I). In einem zweiten Schritt gehen wir auf das Verhältnis von Philologie und Fremdsprachigkeit ein, das sich insbesondere am Problem der Übersetzung und des Fremdwortgebrauchs ablesen lässt (Abschn. II), um dann in einem dritten Schritt die Ausgangsfrage nach Grenzen und Alternativen des Einsprachigkeitsparadigmas in der Philologie wieder aufzugreifen (Abschn. III).

Derrida macht in dem berühmten Satz »Je n'ai qu'une langue, ce n'est pas la mienne« (1996: 13) ${ }^{2}$ darauf aufmerksam, dass der Idiolekt eines jeden von uns ebenso individuell wie uns selbst unverfügbar bleibt. Alles, was wir sprechen, ist in seinen Einheiten und Strukturen von anderen (vor-)geprägt - daran ändert auch das Faktum nichts, dass wir es uns auf eine je singuläre Weise aneignen und $\mathrm{zu}>$ unserer< Sprache zusammenfügen. Die Einsprachigkeit, wie sie das

1 | "[T]here are strong arguments for mother tongue education, for an understanding of multilingualism as the global norm, for understanding the prevalence of code-switching in bi- and multilingual communities, and for the importance of language rights to provide a moral and legal framework for language policies. Our position, however, is that although such arguments may be preferable to blinkered views that take monolingualism as the norm, they nevertheless remain caught within the same paradigm. [...] [M] ltlinlinguism therefore simply becomes a pluralization of monolingualism " (Makoni/Pennycook 2005: 147).

2 | "Ich habe nur eine Sprache, und die ist nicht die meinige." (Derrida 2011: 153) 
Einsprachigkeitsparadigma denkt, befindet sich sowohl jenseits der einmaligen Sprachlichkeit jedes einzelnen Sprechers als auch jenseits der multiplen Sprachlichkeit jeder Kommunikationssituation, in der die Idiolekte in Verhandlung miteinander treten, sich unterschiedliche sprachliche Standards aneinander reiben und die Begrenztheit jeder vereinzelten Sprache sowie die eindeutige Sprachigkeit der verwendeten Strukturen potentiell angetastet wird. ${ }^{3}$ Sprache im Sinne des Einsprachigkeitsparadigmas wäre ein genau abgegrenztes Gemeinschaftseigentum, dem die Unsauberkeiten sowohl des Idiolekts als auch der alltäglichen Kreolisierung fremd sind.

Dennoch: Eine solche Argumentation kann die soziale Wirkmächtigkeit des Einsprachigkeitsparadigmas nur bedingt ankratzen. Das liegt auch daran, dass dieses Paradigma gewissermaßen >nur den ideologischen Überbau zu sprachpolitischen Strategien bildet, die die sprachsoziologischen Gegebenheiten bereits massiv verändert haben. Im Namen der Einsprachigkeit haben zahllose pädagogische und politische Institutionen daran gearbeitet - und arbeiten weiterhin daran -, sprachliche Standards zu erfinden und Abweichungen zu sanktionieren. Die Durchsetzung des Einsprachigkeitsparadigmas ging und geht einher mit der Etablierung von Sprachgrenzen und der Beseitigung sprachlicher Varianzen und Übergangsphänomene. An die Stelle der je singulären Sprache, die der Einzelne als Erstes lernt und die beispielsweise auch die Variante eines lokalen Dialektes sein könnte, wird eine Standardsprache gesetzt, die fortan gleichwohl als Muttersprache zählt. Muttersprache im Sinne des Einsprachigkeitsparadigmas konstituiert sich so paradoxerweise durch die Unterdrückung und Überwindung dessen, was man zuerst (und zumal mit der Mutter) gesprochen hat. Weil das Einsprachigkeitsparadigma glauben macht, dass nicht die Sprache der Mutter, sondern die Standardsprache der Schule die Muttersprache sei, ist eben nicht nur der Dialektsprecher von dieser Unterdrückung bzw. Überwindung des Ursprünglichen betroffen, sondern im Prinzip ein jeder. Noch die sprachpflegerische Rettung >sterbender < Sprachen tendiert dazu, vorgeblich >ethnisch< der Sprachgemeinschaft zugehörigen Sprechern ihre seigentliche< Muttersprache aufzuzwingen. Die Sprachsituation in Luxemburg ist für den alles andere als eindeutigen Status von >Muttersprachigkeit< ein rezentes Beispiel, spiegelt sich doch die Lage Luxemburgs im Kontakt- und Vermittlungsraum zwischen Germania und Romania in einer Situation wider, »die von einem Nebeneinander des Deutschen, bzw. regionaler Varietäten des Deutschen und des Französischen, geprägt ist.« (Gilles u.a. 2010: 63) Die aktuelle Triglossiesituation im Land, d.h. das Nebeneinander des Luxemburgischen, Deutschen und Französischen - führt dann zu der Frage, für wen das durch die 1984 erlassene Sprachgesetzgebung zur Nationalsprache erhobene Luxemburgische nun als Muttersprache fungieren kann und soll und welche Rolle die beiden anderen Landessprachen in diesem Kontext einnehmen.

3 I Im Sinne der Semiotik ist die Interaktion zwischen zwei 'Sprachen، Bedingung der Möglichkeit von Kommunikation (vgl. Lotman 2010: 163ff.). 
Zu Recht verweisen Makoni und Pennycook darauf, dass in solchen Situationen »rights are attached to languages rather than to their speakers« (2005: 149). ${ }^{4}$ Damit aber wurden und werden sprachliche Realitäten geschaffen, deren artifizielle Konstitution indes nicht gegen ihre Beharrlichkeit spricht - und auch nicht gegen ihre Funktionalität, denn es liegt auf der Hand, dass es Tendenzen zur Standardisierung von Sprachnormen immer geben muss, soll überhaupt Aussicht darauf bestehen, dass man Antworten auf sprachliche Äußerungen erhält. ${ }^{5}$ Will man den Befund auf die Spitze treiben, so kann man formulieren, dass die Annahmen des Einsprachigkeitsparadigmas zwar schlicht falsch sind weder gibt es scharfe Sprachgrenzen und strikt eindeutige Sprachigkeit, ${ }^{6}$ noch verfügen Sprecher über eine Muttersprache -, dass aber dennoch Spracheinheiten nicht nur etwas sind, womit man ganz real rechnen muss, wenn man sich mit Sprache beschäftigt, sondern sie in bestimmter Hinsicht auch schon fast eine Art Denknotwendigkeit darstellen.

Ein Stück weit lässt sich dieser Befund durch Unschärfen erklären: Real sind die Einsprachigkeit und die Vielheit der Einsprachigkeiten insofern, als die sogenannten modernen Nationalsprachen effektiv standardisierte Verdichtungen sprachlicher Ausdrucksmöglichkeiten darstellen. Diese Standardisierungen sind erfolgreich, weil sie - in Abhängigkeit von den jeweils zur Verfügung stehenden Medien - die situationsunabhängige Einsetzbarkeit von Sprache gewährleisten. ${ }^{7}$ Wenn sie auch an ihren Grenzen ausfransen, wenn sie auch in sich eine Vielfalt sprachlich >fremder < Strukturen bergen, so lassen sich Wörter, Sätze und Texte doch regelmäßig sehr zuverlässig einzelnen Sprachen zuordnen und werden >Fehler< von unterschiedlichen Sprechern übereinstimmend konstatiert. Ob man also >große< Spracheinheiten im Sinne des Einsprachigkeitsparadigmas sieht oder ein buntes Chaos singulärer Idiolekte, ist auch eine Frage der Tiefenschärfe oder der Betrachtungsebene.

Aber das Problem ist grundsätzlicherer Natur - und zwar nicht zuletzt deshalb, weil das Einsprachigkeitsparadigma in seinen unterschiedlichen Spielarten gegenüber einer solch unscharfen Beschreibung von Sprachstandards auf

4 | Zur Kritik an der ,Öko-Linguistikı vgl. Pennycook 2004.

5 | Hier wäre historisch weiter zu differenzieren, denn selbstverständlich gibt es wirkmächtige Kulturpolitiken, die Sprachstandards durchsetzen, schon sehr lange - beispielsweise mit dem Ziel der Bewahrung heiliger Sprachen. Das Bemerkenswerte an der neuzeitlichen Vorstellung von Einsprachigkeit liegt darin, dass die Vorstellung vorherrscht, Standardsprachen seien gleichwohl in den Muttersprachlern verkörpert. Hierzu, insbesondere zu Herders Konzept der Muttersprache (vgl. Martyn 2013).

6 | Zur Unmöglichkeit der Zählung von Sprachen (und für eine frühe Dekonstruktion des Konzepts 'Muttersprache`) siehe bereits Thümmel 1977. Aus übersetzungstheoretischer Perspektive siehe Sakai 2009. Zum Problem, die Sprachen über Korrektheit abzugrenzen, genauer: zur prinzipiellen Ununterscheidbarkeit von Fehler und (rhetorischer) Figur siehe Martyn 2004.

7 | Die Abhängigkeit unterschiedlicher Sprachstandards von der jeweils tonangebenden Medientechnik analysiert Giesecke (1992: 280-334) anhand der Entwicklung der deutschsprachigen Fachprosa nach der Durchsetzung des Buchdrucks. 
mehr Eindeutigkeit besteht. So soll die Annahme, dass sich Sprachen als geschlossene Ausdruckssysteme gegen Störungen von Außen abdichten können, spätestens seit dem Aufkommen rationalistischer Denkmodelle im 17. und 18. Jahrhundert auch ihre Befähigung zum Ausdruck von Wahrheiten sichern. Der Rationalismus geht in seinen vielen unterschiedlichen Formen unter anderem davon aus, dass Sprache und Denken einander äquivalent und grundsätzlich dazu fähig sind, Welt eindeutig und einheitlich zu erfassen. ${ }^{8}$ Er setzt dementsprechend auf Kommunikationsmodi, die eindeutige Bezeichnungen (Klarheit und Deutlichkeit) sowie Widerspruchsfreiheit einfordern. Ist dies die Maßgabe, so muss jeder Anflug von Idiolekt oder Kreolisierung als Störung, Anomalie oder Rauschen gelten, weil dann die Eindeutigkeit der Bezeichnungen in Frage steht. ${ }^{9}$ Das Bemühen, Sprache so zu kontrollieren, dass sie genau eine Systematik von Ausdrucksmöglichkeiten bereitstellt, ist so gesehen ein Korrelat der rationalistischen Grundannahmen - und diese Grundannahmen gelten zumindest teilweise auch noch für diejenigen wissenschaftlichen Argumentationen, die zu Recht die Abgrenzbarkeit von Spracheinheiten und die übrigen Grundprinzipien des Einsprachigkeitsparadigmas bestreiten. Die Idee der Einsprachigkeit erscheint so gesehen als eine Art Regulativ (im Sinne Kants) all jener Kommunikationsformen, die rationalistische Grundannahmen teilen, insbesondere also vieler Wissenschaften (vgl. Saki 2009: 73f.). Man geht davon aus, dass sie möglich ist, auch wenn derzeit allenfalls die Sprache der Mathematik behaupten kann, die Ansprüche des rationalistischen Kommunikationsideals zu erfüllen.

Für die Sprache der Wissenschaft ist das Regulativ der Einsprachigkeit in den Debatten um den Stellenwert des Englischen thematisiert worden. Das liegt zumal für die gegenwärtige Linguistik und selbst für die Philologie der Mehrsprachigkeit nahe - ist es doch bemerkenswert, dass etwa ein so verdienstvolles Buch wie Yildiz' Beyond the Mother Tongue mit seinem Plädoyer für eine Überwindung der Einsprachigkeit die vielen deutschen Texte, die es behandelt, an vielen Stellen nur in englischer Übersetzung zitiert. Gegen die Vormachtstellung des Englischen hat insbesondere Ehlich seit den 1990er Jahren in vielen Vorträgen die wissenschaftliche Mehrsprachigkeit verteidigt. ${ }^{10}$ Damit wendet er sich gegen die rationalistische Auffassung, der Wissenschaft sei Einsprachigkeit förderlich und es sei im Sinne des wissenschaftlichen Fortschritts vernünftig, sich auf eine Lingua franca zu einigen. Dem ersten Anschein zum Trotz sind Ehlichs Argumente allerdings durch und durch selbst vom Einsprachigkeitsparadigma geprägt. Ehlich geht - in Anlehnung an Gedankengut, das sich von

8 | Zu den 'Axiomen ، des Rationalismus vgl. Bunia 2013: Kap. 1.

9 | Es wäre lohnend, das Erbe des rationalistischen Denkens in der heutigen analytischen Philosophie im Hinblick auf Fragen der Einsprachigkeit genauer zu besehen. Spätestens seit Russels Principia mathematica (1910-1913) steht das Bemühen darum, Argumente auf einheitliche formalsprachliche Strukturen zurückzuführen, im Mittelpunkt ihrer Anstrengungen. Schnädelbach zufolge führt gerade der Übergang in eine postanalytische Phase bei Davidson schließlich in einen "neue[n] Monismus des sprachlichen Umgangs mit der Welt" (Schnädelbach 2004: 399).

10 | Siehe z.B. Ehlich 2005, 2006 u. 2009. 
Herder und Wilhelm von Humboldt her in meist trivialisierter Form durch die Sprachdebatten zieht - von der Vorstellung aus, dass Sprache nicht nur die kulturelle Identität von Sprechern bedingt, sondern auch ein epistemologisches Bedingungsgerüst darstellt. Jede Erkenntnis hängt demzufolge von den Möglichkeiten ab, die die Sprache, in der sie sich vollzieht, ihr zur Verfügung stellt. Damit ist zumal Wissenschaft immer schon grundlegend geprägt von der Sprache, in der sie entwickelt wird - woraus sich für Ehlich das Phänomen nationaler Wissenschaftskulturen erklärt. Der Angriff auf diese Wissenschaftskulturen kommt daher für Ehlich einem Angriff auf die Quellenvielfalt wissenschaftlicher Erkenntnis gleich, insbesondere in den Geisteswissenschaften, in denen ihm zufolge im Finden des rechten sprachlichen Ausdrucks die zentrale Erkenntnisleistung selbst steckt, wohingegen die mathematisierten Wissenschaften eher über die Möglichkeit eines kultur- und sprachunabhängigen Erkenntnisausdrucks verfügen. Begäbe man sich dieser Ausdrucksmöglichkeiten, so hätte dies einerseits eine Verarmung der geisteswissenschaftlichen Erkenntnismöglichkeiten zur Folge, andererseits aber auch eine Verarmung der Nationalsprachen selbst, deren Ausdrucksvielfalt von der Pflege einer Wissenschaftssprache abhängig sei. Folgt man Ehlich, so haben die Wissenschaften für die Durchsetzung nationaler Standardsprachen in der Neuzeit einen entscheidenden Beitrag geleistet. An seiner Argumentation lässt sich aber auch ablesen, dass die Forderung wissenschaftlicher Mehrsprachigkeit das rationalistische Streben nach eindeutiger Spracheinheit nur vervielfältigt - die einzelnen Sprachen sollen jede für sich ihre eindeutige Perspektive auf die Welt entwickeln; sie bleiben aber im Nachhinein, dank ihrer gemeinsamen Orientierung an der Wahrheit, immer ineinander übersetzbar. Nicht zufällig fordert Ehlich eine intensivere wissenschaftliche Übersetzungstätigkeit. ${ }^{11}$

Was bedeutet all dies für diejenigen Wissenschaftsdisziplinen, deren Erbe wir hier und heute antreten, die Nationalphilologien? Man wird zweierlei festhalten können: Zum einen haben sich Fächer wie die Germanistik im Fahrwasser des Nationalismus des 19. Jahrhunderts entwickelt. Die Pflege einer deutschen Wissenschaftssprache gehörte damit selbstverständlich zu ihren zentralen Aufgaben. Zum anderen aber implizierte der Übergang Von der gelehrten zur disziplinären Gemeinschaft, wie der Titel eines einschlägigen Sammelbandes zur Geschichte der Germanistik lautet (Fohrmann/Voßkamp 1987), zunächst den Zwang zur begrifflichen und methodischen Standardisierung, durch die eine situationsunabhängige, allgemeine Adressierbarkeit der Ergebnisse gewährleistet werden sollte, führte dann aber auch immer wieder, wenn man nämlich die eigene Wissenschaftlichkeit unter Beweis stellen wollte, zu einer Kultivierung von

11 | Mit Pennycook (2004) könnte man präzisieren, dass Ehlich Mehrsprachigkeit als Heteroglossie denkt, also als Vielfalt der Möglichkeiten, ein und dasselbe auszudrücken. Im Rückgriff auf dieses Konzept hat Gramling wiederholt darauf hingewiesen, dass das moderne Konzept von Einsprachigkeit unmittelbar an die Vorstellung einer problemlosen Transponierbarkeit von Aussagen in andere Sprachen gebunden ist (siehe z.B. Gramling 2010). 
Eindeutigkeit im Sinne des rationalistischen Kommunikationsideals. Philologie umfasste zunehmend die Herstellung eindeutiger Textbefunde, standardisierter Werkausgaben und festgefügter Traditionszusammenhänge; und darüber hinaus auch die Entwicklung eindeutiger fachsprachlicher Begrifflichkeiten. In diesem doppelten Rahmen bewegen wir uns zumindest teilweise noch hier und heute, wenn wir versuchen, das Problem der Einsprachigkeit zu beschreiben; denn unsere Disziplin leitet ihre Daseinsberechtigung auch davon her, dass sie über präzise Beschreibungssprachen zu verfügen behauptet, die für unsere Gegenstände angeblich besser geeignet sind als andere. ${ }^{12}$

Dennoch: Die Einordnung der Philologie in die Reihe der Wissenschaften mit Einsprachigkeitsideal lässt sich nicht ganz umstandslos vollziehen. Denn in ihren Ursprüngen, historisch gesehen also lange vor der Durchsetzung des Einsprachigkeitsparadigmas, konstituiert sich die Philologie gerade in der Auseinandersetzung mit dem Fremden und schwer Verständlichen; ja der philologische Text im ursprünglichen Sinne gewinnt seine (prekäre) Einheit aus der Begegnung mehrerer Sprachen und aus der Überschreitung von Sprachgrenzen heraus, die in ihnen stattfindet. Man muss also nicht nur fragen, inwiefern die Philologie einem rationalistischen Standard überhaupt je verpflichtet sein kann, sondern auch, welche Alternativen es für die Etablierung wissenschaftssprachlicher Standards gibt.

\section{II}

Die Philologie, so kann man bei Friedrich Nietzsche lesen,

ist jene ehrwürdige Kunst, welche von ihrem Verehrer vor Allem Eins heischt, bei Seite gehn, sich Zeit lassen, still werden, langsam werden -, als eine Goldschmiedekunst und -kennerschaft des Wortes, die lauter feine vorsichtige Arbeit abzuthun hat und Nichts erreicht, wenn sie es nicht in lento erreicht. [...] sie lehrt gut lesen, das heisst langsam, tief, rück- und vorsichtig, mit Hintergedanken, die offen gelassenen Thüren, mit zarten Fingern und Augen lesen [...]. (Nietzsche 1988: 17)

12 Im Einzelnen wäre die Bedeutung der deutschen Sprache für die Entstehung der modernen deutschen Literaturwissenschaft noch zu ergründen. Wissenschaftssoziologisch entscheidend ist die Notwendigkeit, Leitdifferenzen, Methoden und Gegenstände zu etablieren, die für die Disziplin im Gegensatz zu anderen Disziplinen charakteristisch sind. Die Bemühungen dazu verlaufen im Einzelnen im 19. und 20. Jahrhundert relativ komplex und nur teilweise sind die Bezugnahme auf ,Nation, bzw. nationale Erziehung entscheidend. Insgesamt kann man aber vermuten, dass zum einen das rationalistische Paradigma eine tragendende Rolle für die 'Disziplinierung، des Fachs gespielt haben dürfte und dass zum anderen das Nachdenken über die philologische Begriffssprache deren Einsprachigkeit vorausgesetzt haben wird. Vgl. hierzu z.B. Fohrmann 1989 u. Fohrmann/Voßkamp 1994. 
Es erscheint uns ziemlich sicher, dass Nietzsches Verständnis der Philologie weder dem nationalphilologischen Duktus des 19. Jahrhunderts noch der rationalistischen Tradition verpflichtet bleibt. Dem ausgebildeten Alt-Philologen gehörte der Problembereich der Übersetzung gleichsam zum Berufsbild, so dass die eben zitierte Beschreibung der Philologie auch als eine Beschreibung dieses altphilologischen Kerngeschäfts begriffen werden muss. Dass von hier, nämlich von Fragen der Übersetzung aus, ein neues Licht auf das Verständnis des philologischen Tätigkeitsbereichs sowie der Philologie als Disziplin selbst geworfen wird, muss nicht unbedingt überraschen. Die Auseinandersetzung mit dem Fremden, dem Unvertrauten, dem Neuen, also der Umgang mit Sprach- und Verstehensgrenzen ist ja gerade das, was das Geschäft der Interpretation in allen seinen Facetten motiviert.

Allerdings ist die philosophische Hermeneutik, wie sie richtungweisend von Hans-Georg Gadamer in Wahrheit und Methode entfaltet wurde, mit Blick auf die Fragestellung, wie das Fremde in seiner Fremdartigkeit adäquat zu verstehen sei, zumindest dort nicht sehr hilfreich, wo sie dafür plädiert, das Verstehen »selber nicht so sehr als eine Handlung der Subjektivität $z u$ denken, sondern als Einrücken in ein Überlieferungsgeschehen.« (Gadamer 1990: 295; Hervorh. im Orig.) An so einem Überlieferungsgeschehen aber hat das Fremde und damit ja auch und vor allem eine fremde Sprache per definitionem nicht teil, denn Überlieferung besteht hier letztlich in nichts anderem als der beständigen Reproduktion von Verstehensgrenzen - selbst wenn diese dabei verschoben werden. Obwohl, wie Gadamer mit Blick auf die Vorurteilsstruktur des Verstehens betont, alles, »was zum Verstehen verlockt, [...] sich selber schon zuvor in seinem Anderssein zur Geltung gebracht haben « muss (ebd.: 304), bleibt dieses Anderssein bei ihm doch nur Ausgangspunkt einer Bewegung, die das Fremde in das Eigene überführt; bleibt das Verstehen also immer der berüchtigte Vorgang der Verschmelzung solcher nur »vermeintlich für sich seiender Horizonte.« (Ebd.: 311; Hervorh. im Orig.) Obwohl sich der Universalitätsanspruch der hermeneutischen Fragestellung doch gerade von der nachhaltigen Irritation des Fremden herleitet, wird dessen Status im Verstehensvollzug sofort wieder nivelliert - kann nivelliert werden, weil der, die oder das Fremde sich letztlich doch einschreiben muss in sprachliche - und das heißt dann eben: gemeinsame - Kontexte: »Sein, das verstanden werden kann, ist Sprache« (ebd.: 478; Hervorh. im Orig.) - und Sprache kennt als solche Gadamer zufolge offenbar keine unüberwindlichen inneren Grenzen:

Die Unverständlichkeit oder Mißverständlichkeit überlieferter Texte, die sie [die Hermeneutik] ursprünglich auf den Plan gerufen hat, ist nur ein Sonderfall dessen, was in aller menschlichen Weltorientierung als das atopon, das Seltsame begegnet, das sich in den gewohnten Erwartungsordnungen der Erfahrung nirgends unterbringen läßt. Und wie im Fortschritt der Erkenntnis die mirabilia ihre Befremdlichkeit verlieren, sowie sie verstanden worden sind, so löst sich auch jede gelingende Aneignung von Überlieferung in eine neue, eigene Vertraulichkeit auf [...]. Beides fließt zusammen in die eine, Geschichte und Gegenwart umspannende, eigene und miteigene Welt, die im Reden 
der Menschen miteinander ihre sprachliche Artikulation empfängt. (Gadamer 1999: 237)

Angesichts solcher Emphase fragt Jacques Derrida zu Recht, ob wir von dem Fremden, »bevor und damit wir ihn bei uns aufnehmen können« (2001: 21), wirklich verlangen sollen, uns zu verstehen, d.h. unsere Sprache zu sprechen, in allen Bedeutungen dieses Ausdrucks. Denn es liegt auf der Hand, dass derjenige, mit dem man bereits all das teilt, was mit einer Sprache geteilt wird, nicht mehr in dem Sinne als Fremder zu bezeichnen ist. Er wäre ja bereits vertraut, wäre heimisch und auf eine spezifische Art lesbar. Seine Textur würde offen liegen, sein Kode wäre entschlüsselt und seine Bedeutung erschöpft. Er hätte aufgehört, uns in unserer Sprachigkeit zu irritieren.

Nun scheint insbesondere die Geste der Übersetzung nachgerade darauf abzuzielen, solche Irritationen zu nivellieren, denn ihre Intention geht, zumindest vorderhand, auf Eindeutigkeit, auf Transkription des Gesagten und Gemeinten, auf die Überschreitung und Überwindung sprachlicher Grenzen. Allerdings ist das Versprechen von Eindeutigkeit trügerisch und eine Übersetzung, die sich diesem Versprechen verpflichtet, hat nicht nur ein strukturelles Problem, sondern verfehlt auch eine spezifische Dimension von Sprache. In der Geste der Übersetzung muss insofern noch etwas anderes angelegt sein, das wir zunächst anhand des wohl kanonischsten Werks der deutschen Literatur illustrieren wollen, nämlich Goethes Faust I. Dort, gleich zu Beginn, in der Szene Studierzimmer, ist der Teufel - in Gestalt eines Pudels - bereits im Haus und Faust, noch ganz von österlicher Stimmung umfangen, will sich den Herausforderungen der Übersetzung stellen. Vielleicht nicht der schlechteste Augenblick.

Wir sehnen uns nach Offenbarung,

Die nirgends würd'ger und schöner brennt

Als in dem neuen Testament.

Mich drängt's, den Grundtext aufzuschlagen,

Mit redlichem Gefühl einmal

Das heilige Original

In mein geliebtes Deutsch zu übertragen.

Geschrieben steht: Im Anfang war das Wort!

Hier stock' ich schon! Wer hilft mir weiter fort?

Ich kann das Wort so hoch unmöglich schätzen,

Ich muß es anders übersetzen,

Wenn ich vom Geiste recht erleuchtet bin.

Geschrieben steht: Im Anfang war der Sinn.

Bedenke wohl die erste Zeile,

Daß deine Feder sich nicht übereile!

Ist es der Sinn, der alles wirkt und schafft?

Es sollte stehn: Im Anfang war die Kraft!

Doch, auch indem ich dieses niederschreibe,

Schon warnt mich was, daß ich dabei nicht bleibe. 
Mir hilft der Geist! Auf einmal seh' ich Rat

Und schreibe getrost: Im Anfang war die Tat!

Das klägliche Heulen des Pudels im Verlaufe dieser Übersetzungsreflexion zeigt, dass Faust wohl auf dem richtigen Weg ist. Und in der Tat wird in dieser kurzen Szene viel von dem deutlich, was mit Blick auf die Frage der Übersetzung und ihr Verhältnis zum Original von großem Interesse ist und auf das in theoretischer Hinsicht zunächst Walter Benjamin und - ihm in gewisser Weise folgend - dann auch Theodor W. Adorno aufmerksam gemacht haben. Denn indem jede Übersetzung zumindest latent die Frage nach dem Original thematisiert, bringt sie zugleich die Differenz von Original und Übersetzung ins Spiel. Nach Benjamin scheint eben in dieser Differenz zum Original das konstitutive Moment der Übersetzung auf. Denn gerade weil die »Treue in der Übersetzung des einzelnen Wortes« den Sinn fast nie voll wiedergeben kann (Benjamin 1972: 17), liegt die Aufgabe des Übersetzers darin, das Original zu verändern. »Die wahre Übersetzung«, so Benjamin, »ist durchscheinend, sie verdeckt nicht das Original [...], sondern läßt die reine Sprache, wie verstärkt durch ihr eigenes Medium, nur um so voller aufs Original fallen.« (Ebd.: 18) Es geht somit in der Übersetzung nicht um die Rehabilitation eines durch die ursprüngliche Einheit des Originals verbürgten Sinns, sondern darum, durch »Umdichtung zu befreien.«(Ebd.: 19) Damit aber ist die Übersetzung nicht nur von der Aporie zwischen Freiheit und Treue zum Text geprägt, sondern kann, gerade weil sie das Original nur in einem unendlich kleinen Punkt des Sinnes berührt, in einer gleichsam dekonstruktiven Geste die Sprache als Sprachbewegung befreien und auf diese Weise die vermeintlich ursprüngliche Sinneinheit des Originals als Schein demaskieren. Das Original selbst erscheint so als von inneren Sprachgrenzen durchzogen und in seiner Sprachigkeit zutiefst uneindeutig. Daher ist es nur konsequent, wenn die Übersetzungsszene aus Goethes Faust vor Augen führt, dass die adäquate Übersetzung des lateinischen oder griechischen Bibeltextes gerade nicht wortgetreu sich vollziehen kann, sondern eher den Charakter einer (verfremdenden) Umdichtung hat.

Theodor W. Adorno argumentiert in seinem 1959 publizierten Essay Wörter aus der Fremde in eine ganz ähnliche Richtung, wenn er den Fremdwörtern das Potential zuschreibt, »[w]inzige Zellen des Widerstands gegen den Nationalismus « zu bilden (Adorno 2003: 218). ${ }^{13}$ Was an den Fremdwörtern fasziniere, so Adorno, sei eine gewisse »Exogamie der Sprache«, die über den »Umkreis des Immergleichen « hinausweise (ebd.). Dabei nehme das Deutsche - im Gegensatz etwa zum Französischen - deshalb eine Sonderstellung ein, weil hier »die lateinisch-zivilisatorischen Bestandteile nicht mit der älteren Volkssprache verschmolzen, sondern durch Gelehrtenbildung und höfische Sitte eher von jener abgegrenzt wurden.« Weil eben keine Pax romana geschlossen wurde, ist die Deutsche Sprache weniger und mehr zugleich, wie Adorno betont:

13 | Vgl. hierzu Yildiz 2012: 67-108. 
[W]eniger durch jenes Brüchige, Ungehobelte und darum dem einzelnen Schriftsteller so wenig Sicheres Vorgebende, wie es in älteren neuhochdeutschen Texten so kraß hervortritt und heute noch im Verhältnis der Fremdwörter zu ihrer Umgebung; mehr, weil die Sprache nicht gänzlich vom Netz der Vergesellschaftung und Kommunikation eingefangen ist. Sie taugt darum zum Ausdruck, weil sie inn nicht vorweg garantiert. (Ebd.: 220)

Die Fremdwörter stechen in der Deutschen Sprache »unassimiliert heraus«, weshalb ein mit Bedacht gewähltes Fremdwort wie eine silberne Rippe in den Sprachleib eingesetzt werden könne (ebd.: 219). Auf diese Weise tragen Fremdwörter dazu bei, die restaurative Überzeugung, Sprache sei eine organische Einheit oder etwas Naturhaftes, zu überwinden. Die latente Spannung zwischen Fremdwort und >Normalsprache< (und wie wäre die zu bestimmen?) unterbricht das konformistische Moment und die damit einhergehende Beruhigung, Sinn- und Bedeutungszuschreibungen als sicher und stabil und einheitlich zu betrachten. Mit anderen Worten, die Diskrepanz zwischen Fremdwort und Sprache tritt in den Dienst der (anti-rationalistischen) Aufklärung, sie wird Agentin jener Wahrheit, die aufscheint, wenn das immer schon Gewusste als Illusion oder Ideologie entlarvt wird. Doch Adorno geht noch einen Schritt weiter, indem er das Potential des Fremdworts auf das Versprechen der Sprache selbst bezieht. Genau betrachtet nämlich legt das Fremdwort frei, wie es eigentlich um alle Wörter bestellt ist: »daß die Sprache die Sprechenden nochmals einsperrt; daß sie als deren eigenes Medium eigentlich mißlang.« (Ebd.: 221) Dies gilt sowohl für jedes sprachontologische Heilsversprechen wie auch für die Vorstellung, dass Sprache als universaler und universeller Bestandteil einer Kommunikationsgemeinschaft gleichsam eo ipso der Wahrheit verpflichtet ist.

Das universale System der Kommunikation, das scheinbar die Menschen miteinander verbindet und von dem behauptet wird, es sei um ihretwillen da, wird innen aufgezwungen. Nur das Wort, das, ohne auf seine Wirkung zu schielen, sich anstrengt, seine Sache genau zu nennen, hat die Chance, eben dadurch die Sache der Menschen zu vertreten, um die sie betrogen werden, solange jede Sache innen vorgespiegelt wird, als wäre es jetzt, hier die ihre. (Ebd.: 223f.)

Adornos Plädoyer für die Fremdwörter ist letztlich ein Plädoyer für jenes widerständige, schockierende, in sich spannungsvolle Moment der Sprache selbst, das durch keine Übersetzung getilgt, sondern vielmehr in jeder Übersetzung als das Andere und Nichtgesagte der Sprache aufscheinen müsse. Benjamin spricht in Die Aufgabe des Übersetzers von einer »reinen Sprache«, zu der eine gute Übersetzung das Original hin befreien müsse und in der das eigentliche Wesen der Sprache - man könnte ergänzen: jenseits ihrer scheinbar eindeutigen Sprachigkeit - aufscheine. Die Aufgabe des Übersetzers sei es daher, so Benjamin, »[j]ene reine Sprache, die in fremde gebannt ist, in der eigenen $\mathrm{zu}$ erlösen.« (Benjamin 1972-1999: 19) Überraschenderweise kommt Adorno die- 
ser Vorstellung, die bei Benjamin zudem noch messianisch konnotiert wird, erstaunlich nahe, wenn er betont, dass

Fremdwörter etwas von jener Utopie [...] einer Sprache ohne Erde, ohne Gebundenheit an den Bann des geschichtlich Daseienden bewahren [können], die bewußtlos in ihrem kindlichen Gebrauch lebt. Hoffnungslos wie Totenköpfe warten die Fremdwörter darauf, in einer besseren Ordnung erweckt zu werden. (Ebd.: 225)

\section{III}

Die Konfrontation der Verschmelzungsfantasien Gadamers mit Derridas gastrechtlichem Einwand verweist ebenso wie Benjamins und Adornos Ethik des Umgangs mit dem Fremdsprachlichen und mit Sprachgrenzen auf ein weiteres Hindernis auf dem Weg zur Überwindung des Einsprachigkeitsparadigmas. Derridas Hinweis auf die Notwendigkeit, das Fremde als nicht-aneigenbar anzuerkennen, und Adornos Metapher der Fremdwörter als >hoffnungslosen Totenköpfe< zeigen, dass der Einsprachigkeit und der Vielheit der Einsprachigkeiten nicht durch eine Relativierung der grundlegenden Fremdheit der anderen Sprachen beizukommen ist. ${ }^{14}$ Auch wenn das Konzept der Sprachgrenze und der eindeutigen Sprachigkeit sprachlicher Strukturen dekonstruiert ist, sind damit nicht alle Sprachen in Wirklichkeit eine und miteinander >verschmolzen<; vielmehr ist die Vorstellung nicht assimilierbarer sprachlicher Andersartigkeit zu bewahren.

Ein philologisches post-monolinguales Schreiben müsste also das rationalistische Regulativ einer einheitlichen Beschreibungssprache unmissverständlich verabschieden, das der Philologie eigentlich ohnehin immer fremd gewesen ist. Dazu sind mindestens zwei Dinge geboten: zum einen eine Zitierpraxis, die sich die Wiedergabe des sprachlichen Originals und die Problematisierung jeder verwendeten Übersetzung zum Prinzip macht; und zum anderen ein offener Umgang mit >fremdsprachlichen`Begrifflichkeiten, ganz im Sinne des adornoschen Verfahrens. Beide Verfahren dürfen indes keine bloßen Einverleibungen oder Appropriationen darstellen, die die Fremdheit des >fremden Materials antasten - die Integration muss in diesem Sinne >hoffnungslos< bleiben. Man könnte allerdings auch noch einen Schritt weitergehen und Adornos Ausführungen über den Jargon der Eigentlichkeit aufgreifen, die sich zwar auf eine sehr spezifische historische Situation beziehen, in einigen Zügen aber verallgemeinerbar sind. Adorno weist darauf hin, dass sich Jargon durch das Vorherrschen einer bestimmten Anzahl einzelner Wörter auszeichnet, die eine tiefere Bedeutung suggerieren, ohne in den Zusammenhang der Rede wirklich einbezogen zu sein bzw. ohne ob ihrer scheinbaren Bedeutsamkeit in diesen

14 Die sich hier andeutende aporetische Anforderung, die Übersetzung müsse zugleich das Original 'domestizieren، und die Zielsprache 'verfremden', rückt Venuti 1998 ins Zentrum seiner Theorie und Ethik der Übersetzung. 
Zusammenhang einbezogen werden zu müssen (Adorno 1964: 10f., 14 u. 51). Diese Beschreibung verweist nicht nur nebenbei auf ein Risiko des Fremdwortgebrauchs, sondern vor allem darauf, dass Bedeutsamkeit nichts ist, was sich aus der Eindeutigkeit der verwendeten Wörter problemlos ergibt, sondern etwas, was jede Formulierung aufs Neue erarbeiten muss. Ehlichs Behauptung, in den Kulturwissenschaften liege in der jeweiligen Formulierung schon die eigentliche Erkenntnisleistung, lässt sich von hier aus für eine Philologie der Mehrsprachigkeit umdeuten: Wenn der Glaube an die eindeutige Verlässlichkeit der Wörter nur scheinbarer Jargon ist, so muss sich die philologische Formulierung immer schon offen zeigen für die Möglichkeit, auch die scheinbar eindeutige Sprachigkeit der Wörter, die sie verwendet, in Frage zu ziehen. Mindestens drei Strategien post-monolingualen literarischen Schreibens, das genau dies versucht, hat Yildiz in ihren Analysen der Texte Tawadas, Özdamars und Zaimoglus vorgestellt, die vielleicht auch für das philologische Schreiben selbst interessant sind. Für alle drei Autoren heißt post-monolingual zu schreiben, die Kompaktheit der einen Sprache von Innen her zerbrechlich zu machen, insbesondere in der Konfrontation mit anderen Sprachen. Sichtbar wird dabei eine intrinsische Mehrsprachigkeit dieser Sprache, ein Überschuss, der über ihre vermeintlich strikte Grenze hinausweist, ohne dass das >Fremde< dabei geleugnet würde.

Man wird sich allerdings fragen müssen, welche Rückwirkungen solche Verfahren auf die Philologie hätten - und inwiefern die benjaminsche und adornosche Utopie mit den disziplinären Zwänge, denen sich die Philologie aussetzt, kompatibel ist. Eine adäquate Bestimmung des Philologischen, d.h. eine Bestimmung, die dem Rechnung tragen würde, was anhand von Benjamin und Adorno ausgeführt wurde, müsste die Philologie als diejenige Instanz betrachten, welche das Verhältnis der vielen Sprachen in jeder einzelnen Sprache und alle einzelnen Sprachen zueinander nicht bloß beschreibt, sondern vielmehr ist. Philologie wäre dann nichts anderes als »die fortgesetzte Extension der Elemente sprachlicher Existenz « (Hamacher 2010: 2). Dieses Verständnis von Philologie, das den Anforderungen von post-monolingualen Schrift- und Verstehensprozessen zweifelsohne gerecht wird, wirft indes einige systematische Schwierigkeiten auf. Denn eine Idee von Philologie, die sich selbst als das Geschehen der Freilassung der Sprache von der Sprache begreift (ebd.: 49), wird selbst zur Kunst, zu einer ästhetischen Geste ohne methodologischen Kern. Ist das für die Philologie disziplinär verträglich? Kann die Philologie Figuren wie Derrida, der sicherlich als einer der wichtigsten Philologen des 20. Jahrhunderts gelten kann, aber in seiner Schreibweise das im Fach Gängige vielfach gesprengt hat, in höheren Dosen vertragen oder beginnt sie dann, ihre Kontur zu verlieren? Ist für die Philologie das rationalistische Erbe vielleicht doch notwendig, wenn sie als eine Wissenschaft, als Disziplin oder überhaupt als Wissenschaft existieren will? Wir fragen ja nur. Letztlich wird man das ausprobieren müssen. 


\section{LITERATUR}

Adorno, Theodor W. (1964): Jargon der Eigentlichkeit. Zur deutschen Ideologie. Frankfurt a.M.

Ders. (2003): Wörter aus der Fremde [1959]. In: Ders.: Gesammelte Schriften. Hg. v. Rolf Tiedemann. Frankfurt a.M., Bd. XI, S. 216-232.

Benjamin, Walter (1972): Die Aufgabe des Übersetzers [1923]. In: Ders.: Gesammelte Schriften. Unter Mitwirk. v. Theodor W. Adorno u. Gershom Scholem hg. v. Rolf Tiedemann u. Hermann Schweppenhäuser. Frankfurt a.M., Bd. IV, I: Kleine Prosa/ Baudelaire-Übertragungen (hg. v. Tillman Rexroth), S. 9-21.

Bunia, Remigius (2013): Romantischer Rationalismus. Zu Wissenschaft, Politik und Religion bei Novalis. Paderborn.

Derrida, Jacques (1966): Le monolinguisme de l'autre ou la prothèse d'origine. Paris.

Ders. (2001): Von der Gastfreundschaft. Wien.

Ders. (2011): Die Einsprachigkeit des Anderen oder die ursprüngliche Prothese. In: Zeitschrift für interkulturelle Germanistik 2, H. 2, S. 153-168.

Ehlich, Konrad (2005): Deutsch als Medium wissenschaftlichen Arbeitens. In: Markus Motz (Hg.): Englisch oder Deutsch in Internationalen Studiengängen? Frankfurt a.M., S. 41-51.

Ders. (2006): Mehrsprachigkeit in der Wissenschaftskommunikation. In: Ders./Dorothee Heller (Hg.): Die Wissenschaft und ihre Sprachen. Bern u.a., S. 17-36.

Ders. (2009): Deutsch als Wissenschaftssprache für das 21. Jahrhundert. In: Forschung. Politik - Strategie - Management 2, H. 3/4, S. 89-95.

Fohrmann, Jürgen (1989): Das Projekt der deutschen Literaturgeschichte: Entstehung und Scheitern einer nationalen Poesiegeschichtsschreibung zwischen Humanismus und Deutschem Kaiserreich. Stuttgart.

Ders./Voßkamp, Wilhelm (Hg.; 1987): Von der gelehrten zur disziplinären Gemeinschaft. Stuttgart.

Dies. (Hg.; 1994): Wissenschaftsgeschichte der Germanistik im 19. Jahrhundert. Stuttgart/Weimer 1994.

Gadamer, Hans-Georg (1990): Wahrheit und Methode. Grundzüge einer philosophischen Hermeneutik [1960]. 6. Aufl. Tübingen.

Ders. (1999): Rhetorik, Hermeneutik und Ideologiekritik. Metakritische Erörterungen zu Wahrheit und Methode [1971]. In: Ders.: Gesammelte Werke. Tübingen 1999, Bd. 2, S. 232-250.

Giesecke, Michael (1992): Sinnenwandel. Sprachwandel. Kulturwandel. Studien zur Vorgeschichte der Informationsgesellschaft. Frankfurt a.M.

Gilles, Peter u.a. (2010): Sprachen und Identitäten. In: IPSE - Indentités, Politiques, Sociétes, Espaces (Hg.): Doing Identity in Luxemburg. Subjektive Aneignungen - institutionelle Zuschreibungen - sozio-kulturelle Milieus. Bielefeld, S. 63-104.

Gramling, David (2010): On the Other Side of Monolingualism: Fatih Akin's Linguistic Turn(s). In: German Quarterly 83, H. 3, S. 353-372.

Grimm, Jacob (1858): Über den Ursprung der Sprache [1851]. 4. Aufl. Berlin. Hamacher, Werner (2010): 95 Thesen zur Philologie. Frankfurt a.M. 
Lotman, Jurij M. (2010): Die Innenwelt des Denkens. Eine semiotische Theorie der Kultur. Hg. v. Susi K. Frank, Cornelia Ruhe u. Alexander Schmitz. Übers. v. Gabriele Leupold u. Olga Radetzkaja. Frankfurt a.M.

Makoni, Sinfree/Pennycook, Alastair (2005): Disinventing and (Re)Constituting Languages. In: Critical Inquiry in Language Studies. An International Journal 2, S. 137156.

Martyn, David (2004): »«. In: Jürgen Fohrmann (Hg.): Rhetorik. Figuration und Performanz. Stuttgart/Weimar, S. 397-419.

Ders. (2013): Literatur als Zweitsprache von Leibniz bis Tawada. Paderborn [im Druck]. Nietzsche, Friedrich (1988): Morgenröte [1881]. In: Ders.: Sämtliche Werke. Kritische Studienausgabe in $15 \mathrm{Bdn}$. Hg. v. Giorgio Colli u. Mazzino Montinari. München, Bd. 3, S. 9-331.

Pennycook, Alastair (2004): Language Policy and the Ecological Turn. In: Language Policy 3, H. 3, S. 213-239.

Sakai, Naoki (2009): How do we count a language? Translation and discontinuity. In: Translational Studies 2, H. 1, S. 71-88.

Schnädelbach, Herbert (2004): Analytische und postanalytische Philosophie. In: Ders.: Analytische und postanalytische Philosophie. Vorträge und Abhandlungen 4. Frankfurt a.M., S. 9-44.

Stockhammer, Robert/Arndt, Susan/Naguschewski/Dirk (2007): Einleitung. Die Unselbstverständlichkeit der Sprache. In: Dies. (Hg.): Exophonie. Anders-Sprachigkeit (in) der Literatur. Berlin, S. 7-27.

Thümmel, Wolf (1977): Kann man Sprachen zählen? Bemerkungen zu einigen begrifflichen Unterscheidungen bei Harald Haarmann. In: Osnabrücker Beiträge zur Sprachtheorie 4, S. 36-60.

Venuti, Lawrence (1998): The Scandals of Translations. Towards an ethics of difference. London/New York.

Yildiz, Yasemin (2012): Beyond the Mother Tongue. The postmonolingual condition. New York. 
